\title{
Finite Approximations to a Zero-Sum Game With Incomplete Information
}

\author{
By J. W. Mamer ${ }^{1}$ and K. E. Schilling ${ }^{2}$
}

Abstract: In this paper, we investigate a scheme for approximating a two-person zero-sum game $G$ of incomplete information by means of a natural system $G_{m n}$ of its finite subgames. The main question is: For large $m$ and $n$, is an optimal strategy for $G_{m n}$ necessarily an $\epsilon$-optimal strategy for $G$ ?

\section{Introduction}

To formalize our idea of approximating a two-person zero-sum game of incomplete information by its subgames, we introduce what we shall call a game structure. A game structure is a system of the form $\left(\Omega, U, \tilde{F}_{m}, \tilde{G}_{n}\right)_{m, n=1}^{\infty}$. Here $\Omega=(\Omega, \mathscr{B}, P)$ is a probability space, $U=\left(U_{i j}: i=1, \ldots, M ; j=1, \ldots, N\right)$ is a matrix of random variables on $\Omega$ (the payoff matrix), and $\tilde{F}_{m}$ and $\tilde{G}_{n}$ are sub- $\sigma$-fields of the $\sigma$-field $\mathscr{B}$ such that $\tilde{F}_{m+1} \supseteq \tilde{F}_{m}$ and $\tilde{G}_{n+1} \supseteq \tilde{G}_{n}$. We put $\tilde{F}=\tilde{F}_{\infty}=$ the $\sigma$-field generated by $\cup_{m} \tilde{F}_{m}, \tilde{G}=\tilde{G}_{\infty}=$ the $\sigma$-field generated by $\cup_{n} \tilde{G}_{n}$.

For $m, n=1,2, \ldots, \infty$, let $G_{m n}$ be the two-person, zero-sum game in which a strategy for player I is an $\tilde{F}_{m}$-measurable $\alpha: \Omega \rightarrow S^{M}$, and a strategy for player II is a $\tilde{G}_{n}$-measurable $\beta: \Omega \rightarrow S^{N}$. (Here $S^{M}$ is the simplex $\left\{x \in R^{M}: \sum_{i} x_{i}=1\right.$, $\left.x_{i} \geq 0\right\}$.) If player I plays $\alpha$ and player II plays $\beta$, then the payoff to I is $\Gamma(\alpha, \beta)=$ $E\left(\sum_{i j} U_{i j} \alpha_{i} \beta_{j}\right)$. Thus in the game $G_{m n}, \tilde{F}_{m}$ and $\tilde{G}_{n}$ embody the information available to I and II, respectively. If $\tilde{F}_{m}$ and $\tilde{G}_{n}$ are finite, then $G_{m n}$ is a finite approximation to the game $G=G_{\infty \infty}$.

By standard minimax theorems, each game $G_{m n}$ has saddle point. Let $V_{m n}$ denote the value of the game $G_{m n}$ to player I, and let $V=V_{\infty \infty}$.

1 John W. Mamer, Anderson Graduate School of Management, University of California, Los Angeles.

2 Kenneth E. Schilling, Department of Mathematics, University of Michigan-Flint. 
If $\tilde{F}_{m}$ and $\tilde{G}_{n}$ are finite, then the game $G_{m n}$ is, at least in principle, solvable by finite methods. The question we shall study is: To what extent is an optimal strategy for $G_{m n}$ a useful substitute for an optimal strategy for $G$ ? An ideal result along these lines would be

(1) Fix $\epsilon>0$. Suppose that, for $m, n=1,2, \ldots, \alpha^{m n}$ is an optimal strategy for I in $G_{m n}$. Then, for all sufficiently large $m$ and $n, \alpha^{m n}$ is an $\epsilon$-optimal strategy for $I$ in $G$.

As we shall see, (1) is, alas, in general false. The best we can do is a weaker version of (1) (Theorem 1), and a special case of (1) (Theorem 2). We shall state these theorems presently. For a strategy $\alpha$ for player I in the game $G$, let $\operatorname{Val}_{n}(\alpha)=\inf _{\beta}$ $\Gamma(\alpha, \beta)$, where $\beta$ ranges over $\tilde{G}_{n}$-measurable strategies for II. (Thus if $\alpha$ is $\tilde{F}_{m}$-measurable, then $\operatorname{Val}_{n}(\alpha)$ is the value to I of the strategy $\alpha$ in the game $G_{m n}$.) We shall write $\operatorname{Val}_{G}(\alpha)$ for $\operatorname{Val}_{\infty}(\alpha)$.

Theorem 1: For $m, n=1,2, \ldots$, suppose that $\alpha^{m n}$ is an optimal strategy for player I in $G_{m n}$, and that $\tilde{F}_{m}$ and $\tilde{G}_{n}$ are finite $\sigma$-fields. Then $\lim _{m \rightarrow \infty} \lim _{n \rightarrow \rightarrow \infty} \operatorname{Val}_{G}\left(\alpha^{m n}\right)$ $=V$. Moreover, this convergence is uniform in the choices $\alpha^{m n}$ of optimal strategies, i.e., $\lim _{m \rightarrow \infty} \lim _{n \rightarrow \infty} \inf _{\alpha(m, n)} \operatorname{Val}_{G}(\alpha)=V$, where $A(m, n)$ is the set of strategies optimal for player I in $G_{m n}$.

Theorem 2 says that, under an additional hypothesis, (1) does hold. This hypothesis, which we shall call $(M)$, is a version of the "continuity of information" assumption first used in [Milgrom-Weber]. $(M)$ says roughly that the joint probability on $\tilde{F}$ and $\tilde{G}$ is absolutely continuous with respect to the product probability on $\tilde{F} \times \tilde{G}$. A precise statement of $(M)$ will be found in Sec. 2 .

Theorem 2: Assume ( $M$ holds. If, for $m, n=1,2, \ldots, \alpha^{m n}$ is an optimal strategy for I in $G_{m n}$, then $\lim _{m \rightarrow \rightarrow \infty} \operatorname{Val}_{G}\left(\alpha^{m n}\right)=V$, uniformly in the choices $\alpha^{m n}$ of optimal strategies.

$$
n \rightarrow-\infty
$$

\section{Results}

We first present an example which shows that assertion (1) of the introduction does not hold in general.

Example: A game structure in which (1) fails. 
Let $\Omega$ be the interval [0,1] with Lebesgue measure, $M=N=2$, and the payoff $U_{i j}=\left\{\begin{array}{l}1 \text { if } i=j \\ -1 \text { if } i \neq j\end{array}\right.$ for $i, j=1,2$ (indepenent of $\omega$ ). For $m=1,2, \ldots$, let $\tilde{F}_{m}=\tilde{G}_{m}$ $=$ the $\sigma$-field on $[0,1)$ generated by the partition $\left\{\left[(k-1) / 2^{m}, k / 2^{m}\right): k=1,2, . ., 2^{m}\right\}$. Thus $\tilde{F}=\tilde{G}=$ the Borel $\sigma$-field on $\Omega$. It is easy to see that, for all $m$ and $n, V_{m n}$ $=0$, and in $G_{m n}$ the players have the optimal strategies $\alpha_{1}=\alpha_{2}=\beta_{1}=\beta_{2}=1 / 2$, for all $\omega \in \Omega$.

For finite $m>1$, consider the game $G_{m, m-1}$. The strategy $\underline{\alpha}^{m, m-1}$ given by

$$
\begin{aligned}
& \underline{\alpha}_{1}^{m, n-1}=\left\{\begin{array}{l}
1 \text { if } \omega \in\left[(k-1) / 2^{m}, k / 2^{m}\right), k \text { odd } \\
0 \text { otherwise }
\end{array}\right. \\
& \underline{\alpha}_{2}^{m, m-1}=1-\underline{\alpha}_{1}^{m, m-1}
\end{aligned}
$$

is easily seen to be optimal for player I in the game $G_{m, m-1}$, i.e., $\operatorname{Val}_{m-1}\left(\alpha^{m, m-1}\right)=0$. On the other hand, $\operatorname{Val}_{G}\left(\alpha^{m, m-1}\right)=-1 ; \underline{\alpha}^{m, m-1}$ is a very poor strategy for player I in $G$. Thus in any system $\left(\alpha^{m n}: m, n=1,2, \ldots,\right)$ of optimal strategies for player $\mathrm{I}$ in which $\alpha^{m, m-1}=\underline{\alpha}^{m, m-1}$ for all $m>1, \lim _{\substack{m \rightarrow \rightarrow \infty \\ n \rightarrow \rightarrow \infty}} \operatorname{Val}_{G}\left(\alpha^{m n}\right)<V$.

We shall next prove Theorems 1 and 2 . We first require a series of lemmas. Our first lemma is a special case of Theorem 2 of [Blackwell-Dubins].

Lemma 2.1: Let $\left(X_{k}\right)$ be a uniformly bounded sequence of random variables, and suppose that $X_{k} \rightarrow X_{\infty}$ a.s. as $k \rightarrow \rightarrow$. Then $E\left(X_{k} \mid \tilde{G}_{k}\right) \rightarrow E\left(X_{\infty} \mid \tilde{G}\right)$ a.s. as $k-\rightarrow \infty$.

Our second lemma computes $\operatorname{Val}_{n}(\alpha)$.

Lemma 2.2: Fix a strategy $\alpha$ for $\mathrm{I}$ in $G$, and fix $n \in\{1,2, \ldots, \infty\}$. Define the random variable $\xi$ by $: \xi=$ the $j \in\{1,2, \ldots, N\}$ which minimizes $E\left(\sum_{i} U_{i j} \alpha_{i} \mid \tilde{G}_{n}\right)$. In case of a tie, for definiteness, take the least such $j$. Then, for all strategies $\beta$ for II in $G_{\infty n}$,

(i) $E\left(\sum_{i} U_{i \xi} \alpha_{i}\right) \leq \Gamma(\alpha, \beta)$, so

(ii) $\operatorname{Val}_{n}(\alpha)=E\left(\min _{j} E\left(\sum_{i} U_{i j} \alpha_{i} \mid \tilde{G}_{n}\right)\right)$.

Proof: (i) immediately implies (ii), so we prove (i). Let $\beta$ be a strategy for II in $G_{\infty n}$, that is, a $\tilde{G}_{n}$-measurable $\beta: \Omega \rightarrow S^{N}$. Then, since $\sum_{j} \beta_{j}=1$, we have

$$
E\left(\sum_{i} U_{i \xi} \alpha_{i} \mid \tilde{G}_{n}\right) \leq \sum_{j} \beta_{j} E\left(\sum_{i} U_{i j} \alpha_{i} \mid \tilde{G}_{n}\right)=E\left(\sum_{i j} U_{i j} \alpha_{i} \beta_{j} \mid \tilde{G}_{n}\right) \text { a.s. }
$$

since $\beta$ is $\tilde{G}_{n}$-measurable. Taking expected values on both sides yields (i). 
Lemma 2,3: Let $\left(\alpha^{k}\right)$ be a sequence of strategies for I in $G$, and suppose that $\alpha^{k} \rightarrow \alpha$ a.s. as $k-\rightarrow \infty$. Then

(i) for fixed $n=1,2, \ldots, \infty, \quad \operatorname{Val}_{n}\left(\alpha^{k}\right) \rightarrow \operatorname{Val}_{n}(\alpha)$ as $k-\rightarrow \infty$, and

(ii) $\operatorname{Val}_{k}\left(\alpha^{k}\right) \rightarrow \operatorname{Val}_{G}(\alpha)$ as $k \rightarrow \rightarrow \infty$.

Proof: First note that applying (ii) in a system where $\tilde{G}_{n}=\tilde{G}_{n+1}=\ldots=\tilde{G}_{\infty}$ yields (i), so (i) is a special case of (ii). To prove (ii), let $X_{k}=\sum_{i} U_{i j} \alpha_{i}^{k}$ in lemma 2.1; then we have $E\left(\sum_{i} U_{i j} \alpha_{i}^{k} \mid \tilde{G}_{k}\right) \rightarrow E\left(\sum_{i} U_{i j} \alpha_{i} \mid \tilde{G}\right)$ a.s. as $k \rightarrow \infty$, so by dominated convergence $E\left(\min _{j} E\left(\sum_{i} U_{i j} \alpha_{i}^{k} \mid \tilde{G}_{k}\right)\right) \rightarrow E\left(\min _{j} E\left(\sum_{i} U_{i j} \alpha_{i} \mid \tilde{G}\right)\right)$. By lemma 2.2(ii), we are done.

Lemma 2.4: (i) For $m, n=1,2, \ldots, \infty, \lim _{m \rightarrow \infty} V_{m n}=V_{\infty n}$ and $\lim _{n \rightarrow \infty} V_{m n}=V_{m \infty}$.

$$
\text { (ii) } \lim _{\substack{m \rightarrow \infty \\ n \rightarrow \infty}} V_{m n}=V \text {. }
$$

Proof: Fix $n$, and let $\alpha$ be an optimal strategy for $\mathrm{I}$ in $G_{\infty n}$. Now for $m=1,2, \ldots$, put $\alpha^{m}=E\left(\alpha \mid \tilde{F}_{m}\right)$. Thus $\alpha$ is a legal, though likely not optimal, strategy for I in $G_{m n}$. We have

$$
\operatorname{Val}_{n}\left(\alpha^{m}\right) \leq V_{m n} \leq V_{\infty n}
$$

By lemma 2.1, $\alpha^{m} \rightarrow \alpha$ a.s. By lemma 2.3(i), $\lim _{m \rightarrow \infty} \operatorname{Val}_{n}\left(\alpha^{m}\right)=\operatorname{Val}_{n}(\alpha)=$ $V_{\infty n}$. By the inequality directly above, we infer $\lim _{m \rightarrow \infty} V_{m n}=V_{\infty n}$. By symmetry, we also have $\lim _{n \rightarrow \infty} V_{m n}=V_{m \infty}$. for all $m$. This proves (i). Finally, it is easy to see that $V_{m \infty} \leq V_{m n} \leq V_{\infty n}$. Claim (ii) now follows by letting $m, n-\rightarrow \infty$.

We are now ready to prove Theorem 1 .

Proof of Theorem 1: Suppose that $\alpha^{m n}$ is an optimal strategy for player I in $G_{m n}$, for $m, n=1,2, \ldots$. We claim that, for $m=1,2, \ldots, \lim _{n \rightarrow \rightarrow \infty} \operatorname{Val}_{G}\left(\alpha^{m n}\right)=V_{m \infty}$. To prove this, fix $m$. We shall show that every subsequence of the sequence $\operatorname{Val}_{G}\left(\alpha^{m}\right)$ has in turn a subsequence which converges to $V_{m \infty}$. Indeed, since each $\alpha^{m n}$ is $\tilde{F}_{m}$-measurable, $\tilde{F}_{m}$ being a finite $\sigma$-field, by the Bolzano-Weierstrass theorem every subsequence of $\alpha^{m}$ has a (pointwise) convergent subsequence; thus we may assume that $\alpha^{m n} \rightarrow \alpha^{m}$ as $n \rightarrow \infty$. By lemma 2.3(ii), then, $\operatorname{Val}_{n}\left(\alpha^{m n}\right) \rightarrow \operatorname{Val}_{G}\left(\alpha^{m}\right)$ as $n \rightarrow \rightarrow \infty$. By hypothesis, $\operatorname{Val}_{n}\left(\alpha^{m n}\right)=V_{m n}$, so in fact $V_{m n} \rightarrow \operatorname{Val}_{G}\left(\alpha^{m}\right)$ as $n \rightarrow \rightarrow \infty$. Thus by lemma 2.4(i), $\operatorname{Val}_{G}\left(\alpha^{m}\right)=V_{m \infty}$. On the other hand, since $\alpha^{m n}$ $\rightarrow \alpha^{m}$ as $n \rightarrow \infty$, by 2.3(i) we also have $\operatorname{Val}_{G}\left(\alpha^{m n}\right) \rightarrow \operatorname{Val}_{G}\left(\alpha^{m}\right)=V_{m \infty}$. This proves our claim. 
Now by another use of lemma 2.4(i), $\lim _{m \rightarrow \infty} \lim _{n \rightarrow \infty} \operatorname{Val}_{G}\left(\alpha^{m n}\right)=V$. To prove the "moreover" clause in Theorem 1, let $\left(\epsilon_{m n}\right)$ be a sequence of numbers which converges to 0 as $m, n \rightarrow-\infty$. For all $m, n$, note that there exists $\alpha^{m n} \in$ $A(m, n)$ such that $\operatorname{Val}_{G}\left(\alpha^{m n}\right)-\epsilon_{m n} \leq \inf _{\alpha \in A(m, n)} \operatorname{Val}_{G}(\alpha) \leq \operatorname{Val}_{G}\left(\alpha^{m n}\right)$. The "morevoer" clause follows at once.

We now consider Theorem 2 . We must first discuss hypothesis $(M)$.

$\tilde{F} \times \tilde{G}$ is the $\sigma$-field on $\Omega \times \Omega$ generated by sets of the form $S \times T$, where $S$ $\epsilon \tilde{F}$ and $T \epsilon \tilde{G}$. Let $Q$ and $R$ be the probability measures on $(\Omega \times \Omega, \tilde{F} \times \tilde{G})$ defined by

$$
\begin{aligned}
& Q(A)=P(\{\omega:(\omega, \omega) \in A\}) \text { and } \\
& R(A)=\iint\{(\omega, \eta) \in A\} 1 P(d \omega) P(d \eta) \quad \text { for } A \in \tilde{F} \times \tilde{G} .
\end{aligned}
$$

We now state assumption $(M)$.

(M) $Q$ is absolutely continuous with respect to $R$, that is, for all $A \in \tilde{F} \times \tilde{G}$, if $R(A)=0$, then $Q(A)=0$.

Assumption $(M)$ is a version of a hypothesis introduced in [Milgrom-Weber]. It is easy to see that $(M)$ is satisfied either if $\tilde{F}$ and $\tilde{G}$ are indepedent (in which case $Q=R$ ), or if either $\tilde{F}$ or $\tilde{G}$ is atomic.

Lemma 2.5: Suppose $(M)$ is satisfied. Then if $\left(X_{k}\right)$ is a uniformly bounded sequence of $\tilde{F}$-measurable random variables which converges weakly to $X_{\infty}$, and if $Z$ is any bounded random variable, then

i) $E\left(X_{k} Z \mid \tilde{G}\right) \rightarrow E\left(X_{\infty} Z \mid \tilde{G}\right)$ a.s. and

ii) $E\left(X_{k} Z \mid \tilde{G}_{k}\right) \rightarrow E\left(X_{\infty} Z \mid \tilde{G}\right)$ a.s $\quad$ as $k \rightarrow \infty$.

Proof: First note that, since $E\left(E\left(X_{k} Z \mid \tilde{G}\right) \mid \tilde{G}_{k}\right)=E\left(X_{k} Z \mid \tilde{G}_{k}\right)$, by lemma 2.1,(i) implies (ii). Next, note that we may assume without loss of generality that $Z$ is measurable in $\tilde{F} \vee \tilde{G}$ (the $\sigma$-field generated by $\tilde{F} \cup \tilde{G}$ ). This is because $E\left(X_{k} Z \mid \tilde{G}\right.$ ) $=E\left(X_{k} \cdot E(Z \mid \tilde{F} \vee \tilde{G}) \mid \tilde{G}\right)$, so we may replace $Z$ by $E(Z \mid \tilde{F} \vee \tilde{G})$ if necessary. We shall therefore prove (i), assuming that $Z$ is $\tilde{F} \vee \tilde{G}$-measurable.

Since $Z$ is $\tilde{F} \vee \tilde{G}$-measurable, there exist a bounded $\tilde{F}$-measurable random variable $\hat{X}$, a bounded $\tilde{G}$-measurable random variable $\hat{Y}$, and a bounded, Borel measurable function $f: \mathbf{R} \times \mathbf{R} \rightarrow \mathbf{R}$ such that $Z=f(\hat{X}, \hat{Y})$.

By $(M)$ and the Radon-Nikodym theorem, there exists a bounded function $g: \Omega \times \Omega \rightarrow \mathbf{R}$ such that, for all $A \in \tilde{F} \times \tilde{G}, P(\{\omega:(\omega, \omega) \in A\})=\iint_{A}$ $g(\omega, \eta) P(d \omega) P(d \eta)$. It follows by standard methods that, for any vector $\mathbf{X}$ of $\tilde{F}$ measurable random variables, any vector $\mathbf{Y}$ of $\tilde{G}$-measurable random variables, and any Borel-measurable $h: \mathbf{R}^{p} \rightarrow \mathbf{R}$, we have 
$\left(^{*}\right) E(h(\mathbf{X}, \mathbf{Y}) \mid \tilde{G})(\eta)=\int_{\Omega} h(\mathbf{X}(\omega), \mathbf{Y}(\eta)) g(\omega, \eta) P(d \omega) \quad$ a.s. $[\eta]$.

Now by $(*)$ we have

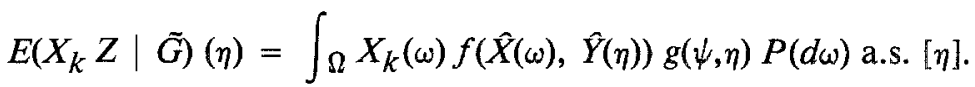

Since, by assumption, $X_{k} \rightarrow X_{\infty}$ weakly, we have $E\left(X_{k} Z \mid \tilde{G}\right)(\eta) \rightarrow-E\left(X_{\infty} Z\right.$ $\tilde{G})(\eta)$ a.s., as desired.

In exact analogy to lemma 2.3 , we have

Lemma 2.6: Assume that $(M)$ holds. If $\left(\alpha^{k}\right)$ is a sequence of strategies for $I$ in $G$ which converges weakly to a strategy $\alpha$, then

(i) for fixed $n=1,2, \ldots, \infty, \operatorname{Val}_{n}\left(\alpha^{k}\right) \rightarrow \operatorname{Val}_{n}(\alpha), \quad$ and

(ii) $\operatorname{Val}_{k}\left(\alpha^{k}\right) \rightarrow \operatorname{Val}_{G}(\alpha) \quad$ as $k \rightarrow \infty$.

Proof of Theorem 2: Suppose that $\alpha^{m n}$ is an optimal strategy for player I in $G_{m n}$, for all finite $m$ and $n$. We shall prove that every sequence $\left(m_{k}, n_{k}\right)$ of pairs of integers such that $m_{k} \rightarrow \infty$ and $n_{k} \rightarrow \infty$ has a subsequence $\left(m_{k}^{\prime}, n_{k}^{\prime}\right)$ such that $\operatorname{Val}_{G}\left(\alpha^{m_{k}^{\prime}}, n_{k}^{\prime}\right) \rightarrow V$ as $k \rightarrow \infty$. To conserve notation, let us write $\alpha^{k}$ for

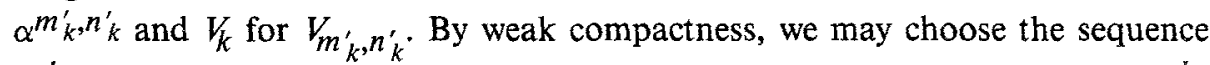
$\left(\alpha^{k}\right)$ to converge weakly to a strategy $\alpha$ as $k \rightarrow \infty$. By lemma 2.6(ii), $\operatorname{Val}_{n_{k}^{\prime}}\left(\alpha^{k}\right)$ $\rightarrow \operatorname{Val}_{G}(\alpha)$ as $k \rightarrow \infty$. By assumption, $\operatorname{Val}_{n_{k}^{\prime}}\left(\alpha^{k}\right)=V_{k}$, and by lemma 2.4 (ii), $V_{k} \rightarrow V$; thus $\operatorname{Val}_{G}(\alpha)=V$.

On the other hand, since $\left(\alpha^{k}\right)$ converges weakly to $\alpha$, by lemma 2.6(i), $\operatorname{Val}_{G}\left(\alpha^{k}\right)$ $\rightarrow \operatorname{Val}_{G}(\alpha)=V$ as $k \rightarrow \infty$. Uniformity follows just as in the proof of Theroem 1. This completes the proof of Theorem 2 .

\section{References}

Blackwell D, Dubins L (1962) Merging of Opinions with Increasing Information, Annals of Mathematical Statistics 33: 882-886

Milgrom P, Weber R (1985) Distributional Strategies for Games with Incomplete Information, Mathematics of Operations Research 10: 619-632

Sion M (1958) On General Minimax Theorems, Pacific Journal of Mathematics 8: 171-176

Received August 1985

Revised version June 1988

Final version November 1989 